\title{
Vectorized Matlab codes for linear two-dimensional elasticity
}

\author{
Jonas Koko \\ LIMOS, Université Blaise Pascal, CNRS UMR 6158, and ISIMA, Campus des Cézeaux, BP 10125, 63173 Aubière \\ Cedex, France \\ E-mail: koko@isima.fr
}

\begin{abstract}
A vectorized Matlab implementation for the linear finite element is provided for the two-dimensional linear elasticity with mixed boundary conditions. Vectorization means that there is no loop over triangles. Numerical experiments show that our implementation is more efficient than the standard implementation with a loop over all triangles.
\end{abstract}

Keywords: Finite element method, elasticity, Matlab

\section{Introduction}

Matlab is nowadays a widely used tool in education, engineering and research and becomes a standard tool in many areas. But Matlab is a matrix language, that is, Matlab is designed for vector and matrix operations. For the best performance in large scale problems, one should take advantage of this.

We propose a Matlab implementation of the $P_{1}$ finite element for the numerical solutions of two-dimensional linear elasticity problems. Instead of mixing finite element types (e.g. [1,2], we propose a $P_{1}$-triangle vectorized code able to treat medium size meshes in "acceptable" time. Vectorization means that there is no loop over triangles nor nodes. Our implementation needs only Matlab basic distribution functions and can be easily modified and refined.

The paper is organized as follows. The model problem is described in Section 2, followed by a finite element discretization in Section 3. The data representation used in Matlab programs is given in Section 4. The heart of the paper is the assembling functions of the stiffness matrix in Section 5 and the right-hand side in Section 6. Numerical experiments are carried out in Section 7 where post-processing functions are given. Matlab programs used for numerical experiments are given in the appendix.

\section{Model problem}

We consider an elastic body which occupies, in its reference configuration, a bounded domain $\Omega$ in $\mathbb{R}^{2}$ with a boundary $\Gamma$. Let $\left\{\Gamma_{D}, \Gamma_{N}\right\}$ be a partition of $\Gamma$ with $\Gamma_{N}$ possibly empty. We assume Dirichlet conditions on $\Gamma_{D}$ and Neumann conditions on $\Gamma_{N}$. Let $u=\left(u_{1}, u_{2}\right)$ be the two-dimensional displacement field of the elastic body. Under the small deformations assumption, constitutive equations are

$$
\begin{aligned}
\sigma_{i j}(u) & =2 \mu \epsilon_{i j}(u)+\lambda \operatorname{tr}(\epsilon(u)) \mathbb{I}_{2}, \quad i, j=1,2, \\
\epsilon(u) & =\left(\nabla u+\nabla u^{T}\right) / 2
\end{aligned}
$$


where $\lambda$ and $\mu$ denote Lamé (positive) constants. These coefficients are related (in plane deformations) to the Young modulus $E$ and the Poisson coefficient $\nu$ by

$$
\lambda=\frac{\nu E}{(1+\nu)(1-2 \nu)}, \quad \mu=\frac{E}{2(1+\nu)} .
$$

Given $f=\left(f_{1}, f_{2}\right) \in L^{2}(\Omega), g=\left(g_{1}, g_{2}\right) \in L^{2}(\Gamma)$ and $u_{D}$, the problem studied in this paper can be formulated as follows

$$
\begin{aligned}
-\operatorname{div} \sigma(u) & =f, \quad \text { in } \Omega, \\
\sigma(u) \cdot n & =g, \quad \text { on } \Gamma_{N}, \\
u & =u_{D}, \quad \text { on } \Gamma_{D} .
\end{aligned}
$$

Let us introduce subspaces

$$
\begin{aligned}
V & =\left\{v \in H^{1}(\Omega): v=0 \text { on } \Gamma_{D}\right\}, \\
V^{D} & =\left\{v \in H^{1}(\Omega): v=u_{D} \text { on } \Gamma_{D}\right\} .
\end{aligned}
$$

The variational formulation of Eqs (2)-(4) is

Find $u \in V^{D}$ such that

$$
\int_{\Omega} \epsilon(u): \mathbb{C} \epsilon(v) d x=\int_{\Omega} f \cdot v d x+\int_{\Gamma_{N}} g \cdot v d s, \quad \forall v \in V .
$$

In Eq. (5), $\mathbb{C}=\left(\mathbb{C}_{i j k l}\right)$ is the fourth-order elastic moduli tensor corresponding to Eq. (1), i.e.

$$
\sigma_{i j}(u)=\sum_{k, l=1}^{2} \mathbb{C}_{i j k l} \epsilon_{i j}(u), \quad i, j=1,2,
$$

where

$$
\mathbb{C}_{i j k l}=\lambda \delta_{i j} \delta_{k l}+\mu\left(\delta_{i k} \delta_{j l}+\delta_{i l} \delta_{j k}\right), \quad 1 \leqslant i, j, k, l \leqslant 2,
$$

$\delta_{i j}$ being the Kronecker delta.

\section{Finite element discretization}

Let $\mathrm{T}_{h}$ be a regular (in the sense of Ciarlet [3]) triangulation of $\Omega$. Spaces $V$ and $V^{D}$ are then replaced by their discrete approximations $V_{h}$ and $V_{h}^{D}$ defined by

$$
\begin{aligned}
V_{h} & =\left\{v_{h} \in \mathrm{C}^{0}(\bar{\Omega}) ; v_{\mid T} \in \mathbb{P}_{1}(T), \forall T \in \mathrm{T}_{h} ; v_{h \mid \Gamma_{D}}=0\right\}, \\
V_{h}^{D} & =\left\{v_{h} \in \mathrm{C}^{0}(\bar{\Omega}) ; v_{\mid T} \in \mathbb{P}_{1}(T), \forall T \in \mathrm{T}_{h} ; v_{h \mid \Gamma_{D}}=u_{D}\right\},
\end{aligned}
$$

where $\mathbb{P}_{1}(T)$ is the space of polynomials of degree less or equals to 1 on the triangle $T$. The discrete version of Eq. (5) is then

Find $u_{h} \in V_{h}^{D}$ such that

$$
\int_{\Omega} \epsilon\left(u_{h}\right): \mathbb{C} \epsilon\left(v_{h}\right) d x=\int_{\Omega} f \cdot v_{h} d x, \quad \forall v_{h} \in V_{h} .
$$

Let $\left\{\phi^{j}\right\}$ be the system of piecewise global basis functions of $V_{h}$, i.e. for all $u_{h}=\left(u_{1 h}, u_{2 h}\right) \in V_{h}$

$$
u_{\alpha h}=\sum_{j=1}^{N} \phi^{j}(x) u_{\alpha}^{j}, \quad \alpha=1,2 .
$$


We set $U=\left(u_{1}^{1} u_{2}^{1} u_{1}^{2} u_{2}^{2} \ldots u_{1}^{N} u_{2}^{N}\right)$, where $u_{\alpha}^{j}$ are nodal values of $u_{h}$, i.e. $u_{\alpha h}\left(x_{j}\right)=u_{\alpha}^{j}$. Applying the standard Galerkin method to Eq. (6) yields

$$
\sum_{j=1}^{N}\left[\int_{\Omega} \epsilon\left(\phi_{i}\right): \mathbb{C} \epsilon\left(\phi_{j}\right) d x\right] U_{j}=\int_{\Omega} f \cdot \phi_{i} d x+\int_{\Gamma_{N}} g \cdot \phi_{i} d s, \quad i=1, \ldots, N .
$$

The stiffness matrix $A=\left(A_{i j}\right)$ and the right-hand side $b=\left(b_{i}\right)$ are then given by

$$
\begin{aligned}
A_{i j} & =\int_{\Omega} \epsilon\left(\phi_{i}\right): \mathbb{C} \epsilon\left(\phi_{j}\right) d x \\
b_{i} & =\int_{\Omega} f \cdot \phi_{i} d x+\int_{\Gamma_{N}} g \cdot \phi_{i} d s .
\end{aligned}
$$

The stiffness matrix $A$ is sparse, symmetric and positive semi-definite before incorporating the boundary condition $u_{\mid \Gamma_{D}}=u_{D}$.

In practice, integrals in Eq. (6) are computed as sums of integrals over all triangles, using the fact that $\bar{\Omega}=\cup_{T \in \mathrm{T}_{h}} T$

$$
\sum_{T \in \mathrm{T}_{h}} \int_{T} \epsilon\left(u_{h}\right): \mathbb{C} \epsilon\left(v_{h}\right) d x=\sum_{T \in \mathrm{T}_{h}} \int_{T} f \cdot v_{h} d x+\sum_{E \subset \Gamma_{N}} \int_{\Gamma_{N}} g \cdot v_{h} d s, \quad \forall v_{h} \in V_{h} .
$$

Let $\left\{\varphi_{i}\right\}$ be the linear basis functions of the triangle $T$ or the edge $E$. If we set

$$
\begin{aligned}
A_{i j}^{(T)} & =\int_{T} \epsilon\left(\varphi_{i}\right): \mathbb{C} \epsilon\left(\varphi_{j}\right) d x \\
b_{i}^{(T)} & =\int_{T} f \cdot \varphi_{i} d x, \\
b_{i}^{(E)} & =\int_{E} g \cdot \varphi_{i} d s,
\end{aligned}
$$

then assembling operations consist of

$$
\begin{aligned}
A_{i j} & =\sum_{T \in \mathrm{T}_{h}} A_{i j}^{(T)} \\
b_{i} & =\sum_{T \in \mathrm{T}_{h}} b_{i}^{(T)}+\sum_{E \subset \Gamma_{N}} b_{i}^{(E)},
\end{aligned}
$$

\section{Data representation of the triangulation}

For the mesh, we adopt the data representation used in Matlab PDE Toolbox [6]. Nodes coordinates and triangle vertices are stored in two arrays $p(1: 2,1: n p)$ and $t(1: 3,1: n t)$, where $n p$ is the number of nodes and $n t$ the number of triangles. The array $t$ contains for each element the node numbers of the vertices numbered anti-clockwise. For the triangulation of Fig. 1, the nodes array $p$ is $(n p=9)$
$0.0000 \quad 1.0000$
1.00000 .0 .5000
1.0000
0.5000
0.0000
0.5000
1.0000
1.0000
0.0000
0.5000
0.0000
0.5000
0.5000

and the elements array $t$ is $(n t=8)$

$\begin{array}{llllllll}5 & 6 & 7 & 8 & 2 & 3 & 4 & 1 \\ 1 & 2 & 3 & 4 & 5 & 6 & 7 & 8 \\ 9 & 9 & 9 & 9 & 9 & 9 & 9 & 9\end{array}$

Neumann boundary nodes are provided by an array ibcneum $(1: 2,1: \mathrm{nbcn})$ containing the two node numbers which bound the corresponding edge on the boundary. Then, a sum over all edges $E$ results in a loop over all entries of ibcneum. Dirichlet boundary conditions are provided by a list of nodes and a list of the corresponding prescribed boundary values.

Note that Matlab supports reading data from files in ASCII format (Matlab function load) and there exists good mesh generators written in Matlab, see e.g. [7]. 


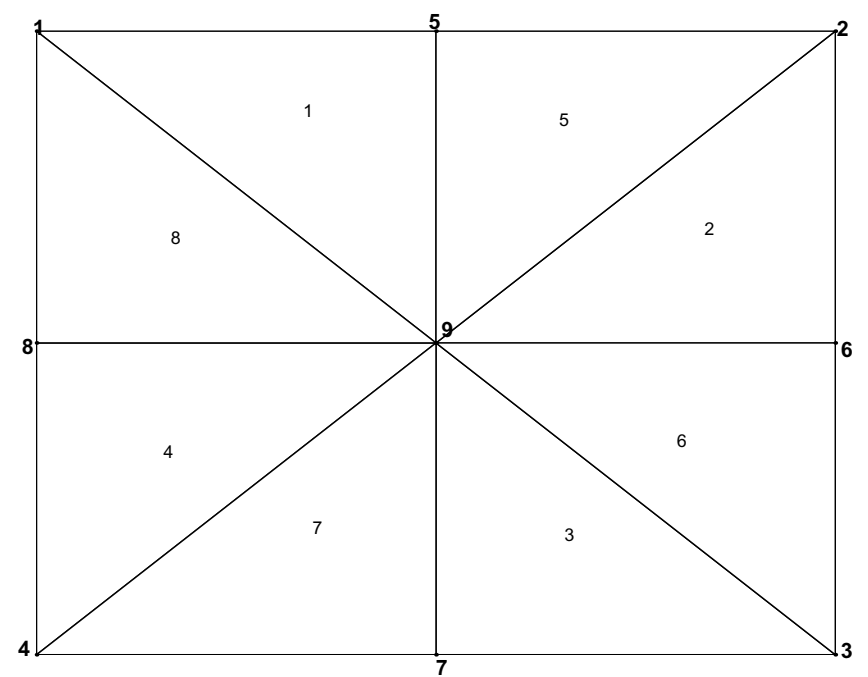

Fig. 1. Plot of a triangulation.

\section{Assembling the stiffness matrix}

As in $[2,5]$ we adopt Voigt's representation of the linear strain tensor

$$
\gamma(u)=\left[\begin{array}{c}
\partial u_{1} / \partial x \\
\partial u_{2} / \partial y \\
\partial u_{1} / \partial y+\partial u_{2} / \partial x
\end{array}\right]=\left[\begin{array}{c}
\epsilon_{11}(u) \\
\epsilon_{22}(u) \\
2 \epsilon_{12}(u)
\end{array}\right] .
$$

Using this representation, the constitutive equation $\sigma\left(u_{h}\right)=\mathbb{C} \epsilon\left(u_{h}\right)$ becomes

$$
\left[\begin{array}{l}
\sigma_{11}\left(u_{h}\right) \\
\sigma_{22}\left(u_{h}\right) \\
\sigma_{12}\left(u_{h}\right)
\end{array}\right]=C \gamma\left(u_{h}\right)
$$

where

$$
C=\left[\begin{array}{ccc}
\lambda+2 \mu & \lambda & 0 \\
\lambda & \lambda+2 \mu & 0 \\
0 & 0 & \mu
\end{array}\right]
$$

Let $\mathbf{u}=\left(u_{1} u_{2} \cdots u_{6}\right)^{t}$ be the vector of nodal values of $u_{h}$ on a triangle $T$. Elementary calculations provide, on a triangle $T$,

$$
\gamma\left(u_{h}\right)=\frac{1}{2|T|}\left[\begin{array}{cccccc}
\varphi_{1, x} & 0 & \varphi_{2, x} & 0 & \varphi_{3, x} & 0 \\
0 & \varphi_{1, y} & 0 & \varphi_{2, y} & 0 & \varphi_{3, y} \\
\varphi_{1, y} & \varphi_{1, x} & \varphi_{2, y} & \varphi_{2, x} & \varphi_{3, y} & \varphi_{3, x}
\end{array}\right]\left[\begin{array}{c}
u_{1} \\
\vdots \\
u_{6}
\end{array}\right]=: \frac{1}{2|T|} R \mathbf{u}
$$

where $|T|$ is the area of the triangle $T$. From Eqs (11) and (12), it follows that

$$
\epsilon\left(v_{h}\right): \mathbb{C} \epsilon\left(u_{h}\right)=\gamma\left(v_{h}\right)^{t} C \gamma\left(u_{h}\right)=\frac{1}{4|T|^{2}} \mathbf{v}^{t} R^{t} C R \mathbf{u}
$$

The element stiffness matrix is therefore

$$
A^{(T)}=\frac{1}{4|T|} R^{t} C R
$$

The element stiffness matrix Eq. (13) can be computed simultaneously for all indices using the fact that 


$$
\left[\begin{array}{l}
\nabla \varphi_{1}^{t} \\
\nabla \varphi_{2}^{t} \\
\nabla \varphi_{3}^{t}
\end{array}\right]=\left[\begin{array}{ccc}
1 & 1 & 1 \\
x_{1} & x_{2} & x_{3} \\
y_{1} & y_{2} & y_{3}
\end{array}\right]^{-1}\left[\begin{array}{ll}
0 & 0 \\
1 & 0 \\
0 & 1
\end{array}\right]=\frac{1}{2|T|}\left[\begin{array}{l}
y_{2}-y_{3} x_{3}-x_{2} \\
y_{3}-y_{1} x_{1}-x_{3} \\
y_{1}-y_{2} x_{2}-x_{1}
\end{array}\right]
$$

Matlab implementation given in [2,5] (in a more modular form) can be summarized by the Matlab Function 5. This implementation, directly derived from compiled languages as Fortran or $\mathrm{C} / \mathrm{C}++$, produces a very slow code in Matlab for large size meshes due to the presence of the loop for. Our aim is to remove this loop by reorganizing calculations.

Function 1. Assembly of the stiffness matrix with the standard loop over triangles

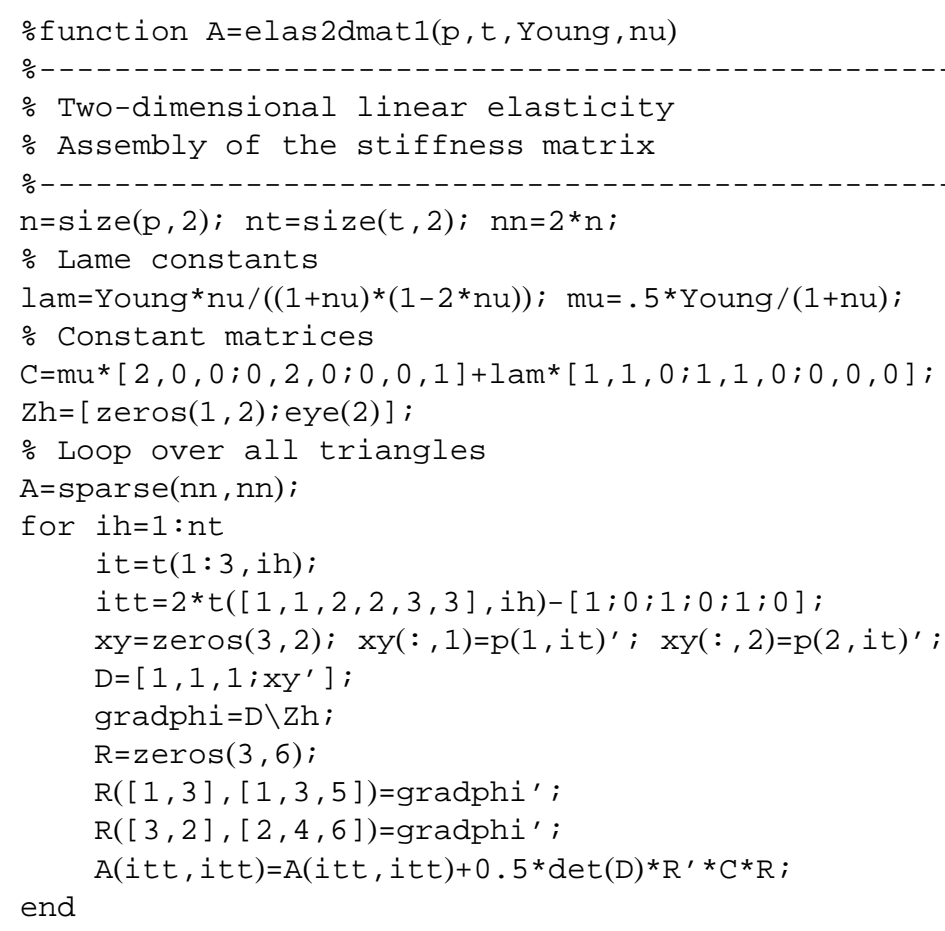

Let us introduce the following notations

$$
x_{i j}=x_{i}-x_{j}, \quad y_{i j}=y_{i}-y_{j}, \quad i, j=1,2,3,
$$

so that, from Eq. (12)

$$
R=\left[\begin{array}{cccccc}
y_{23} & 0 & y_{31} & 0 & y_{12} & 0 \\
0 & x_{32} & 0 & x_{13} & 0 & x_{21} \\
x_{32} & y_{23} & x_{13} & y_{31} & x_{21} & y_{12}
\end{array}\right]
$$

If we rewrite $\mathbf{u}$ in the following non standard form $\mathbf{u}=\left(u_{1} u_{3} u_{5} u_{2} u_{4} u_{6}\right)^{t}$ then the element stiffness matrix can be rewritten as

$$
A^{(T)}=\left[\begin{array}{cc}
A_{11}^{(T)} & A_{12}^{(T)} \\
A_{21}^{(T)} & A_{22}^{(T)}
\end{array}\right]
$$

where $A_{11}^{(T)}$ and $A_{22}^{(T)}$ are symmetric and $A_{21}^{(T)}=\left(A_{21}^{(T)}\right)^{t}$. After laborious (but elementary) calculations, we get 


$$
\begin{gathered}
A_{11}^{(T)}=\left[\begin{array}{ccc}
\tilde{\lambda} y_{23}^{2}+\mu x_{32}^{2} & \widetilde{\lambda} y_{23} y_{31}+\mu x_{32} x_{13} & \widetilde{\lambda} y_{23} y_{12}+\mu x_{32} x_{21} \\
\tilde{\lambda} y_{31} y_{23}+\mu x_{13} x_{32} & \widetilde{\lambda} y_{31}^{2}+\mu x_{13}^{2} & \widetilde{\lambda} y_{31} y_{12}+\mu x_{13} x_{21} \\
\widetilde{\lambda} y_{12} y_{23}+\mu x_{21} x_{32} & \widetilde{\lambda} y_{12} y_{31}+\mu x_{21} x_{13} & \widetilde{\lambda} y_{12}^{2}+\mu x_{21}^{2}
\end{array}\right] \\
A_{22}^{(T)}=\left[\begin{array}{ccc}
\tilde{\lambda} x_{32}^{2}+\mu y_{23}^{2} & \widetilde{\lambda} x_{32} x_{13}+\mu y_{23} y_{31} & \widetilde{\lambda} x_{32} x_{21}+\mu y_{23} y_{12} \\
\widetilde{\lambda} x_{13} x_{32}+\mu y_{31} y_{23} & \widetilde{\lambda} x_{13}^{2}+\mu y_{31}^{2} & \widetilde{\lambda} x_{13} x_{21}+\mu y_{31} y_{12} \\
\tilde{\lambda} x_{21} x_{32}+\mu y_{12} y_{23} & \widetilde{\lambda} x_{21} x_{13}+\mu y_{12} y_{31} & \widetilde{\lambda} x_{21}^{2}+\mu y_{12}^{2}
\end{array}\right]
\end{gathered}
$$

where $\widetilde{\lambda}=\lambda+2 \mu$; and

$$
A_{12}^{(T)}=\left[\begin{array}{l}
\lambda y_{23} x_{32}+\mu x_{32} y_{23} \lambda y_{23} x_{13}+\mu x_{32} y_{31} \lambda y_{23} x_{21}+\mu x_{32} y_{12} \\
\lambda y_{31} x_{32}+\mu x_{13} y_{23} \lambda y_{31} x_{13}+\mu x_{13} y_{31} \lambda y_{31} x_{21}+\mu x_{13} y_{12} \\
\lambda y_{12} x_{32}+\mu x_{21} y_{23} \lambda y_{12} x_{13}+\mu x_{21} y_{31} \lambda y_{12} x_{21}+\mu x_{21} y_{12}
\end{array}\right] .
$$

Let us introduce the following vectors

$$
\mathbf{x}=\left[\begin{array}{l}
x_{32} \\
x_{13} \\
x_{21}
\end{array}\right] \quad \mathbf{y}=\left[\begin{array}{l}
y_{23} \\
y_{31} \\
y_{12}
\end{array}\right]
$$

One can verify that matrices $A_{11}^{(T)}, A_{22}^{(T)}$ and $A_{12}^{(T)}$ can be rewritten in a simple form, using $\mathbf{x}$ and $\mathbf{y}$, as

$$
\begin{aligned}
& A_{11}^{(T)}=(\lambda+2 \mu) \mathbf{y} \mathbf{y}^{t}+\mu \mathbf{x} \mathbf{x}^{t} \\
& A_{22}^{(T)}=(\lambda+2 \mu) \mathbf{x} \mathbf{x}^{t}+\mu \mathbf{y y}^{t} \\
& A_{12}^{(T)}=\lambda \mathbf{y} \mathbf{x}^{t}+\mu \mathbf{x} \mathbf{y}^{t}
\end{aligned}
$$

that is, for $1 \leqslant i, j \leqslant 3$

$$
\begin{aligned}
& A_{11 i j}^{(T)}=(\lambda+2 \mu) \mathbf{y}_{i} \mathbf{y}_{j}+\mu \mathbf{x}_{i} \mathbf{x}_{j}, \\
& A_{22 i j}^{(T)}=(\lambda+2 \mu) \mathbf{x}_{i} \mathbf{x}_{j}+\mu \mathbf{y}_{i} \mathbf{y}_{j}, \\
& A_{12 i j}^{(T)}=\lambda \mathbf{y}_{i} \mathbf{x}_{j}+\mu \mathbf{x}_{i} \mathbf{y}_{j}
\end{aligned}
$$

With Matlab, $\mathbf{x}_{i}$ and $\mathbf{y}_{i}$ can be computed in a fast way for all triangles using vectorization. Then assembling the stiffness matrix reduces to two constant loops for computing $\mathbf{x x}^{t}, \mathbf{y} \mathbf{y}^{t}$ and $\mathbf{x y} \mathbf{y}^{t}$. We do not need to assemble separately $A_{11}^{(T)}, A_{22}^{(T)}$ and $A_{12}^{(T)}$. Sub matrices Eqs (15)-(17) are directly assembled in the global stiffness matrix, in its standard form, since we know their locations. Matlab vectorized implementation of the assembly of the stiffness matrix is presented in Function 2.

Function 2. Assembly of the stiffiness matrix with a vectorized code

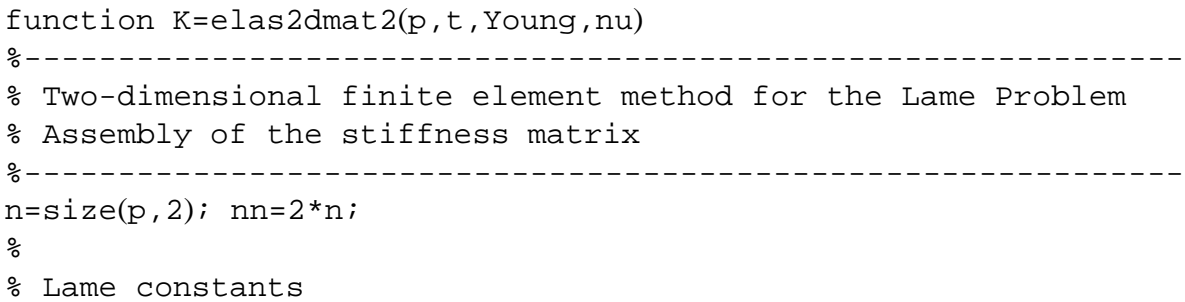




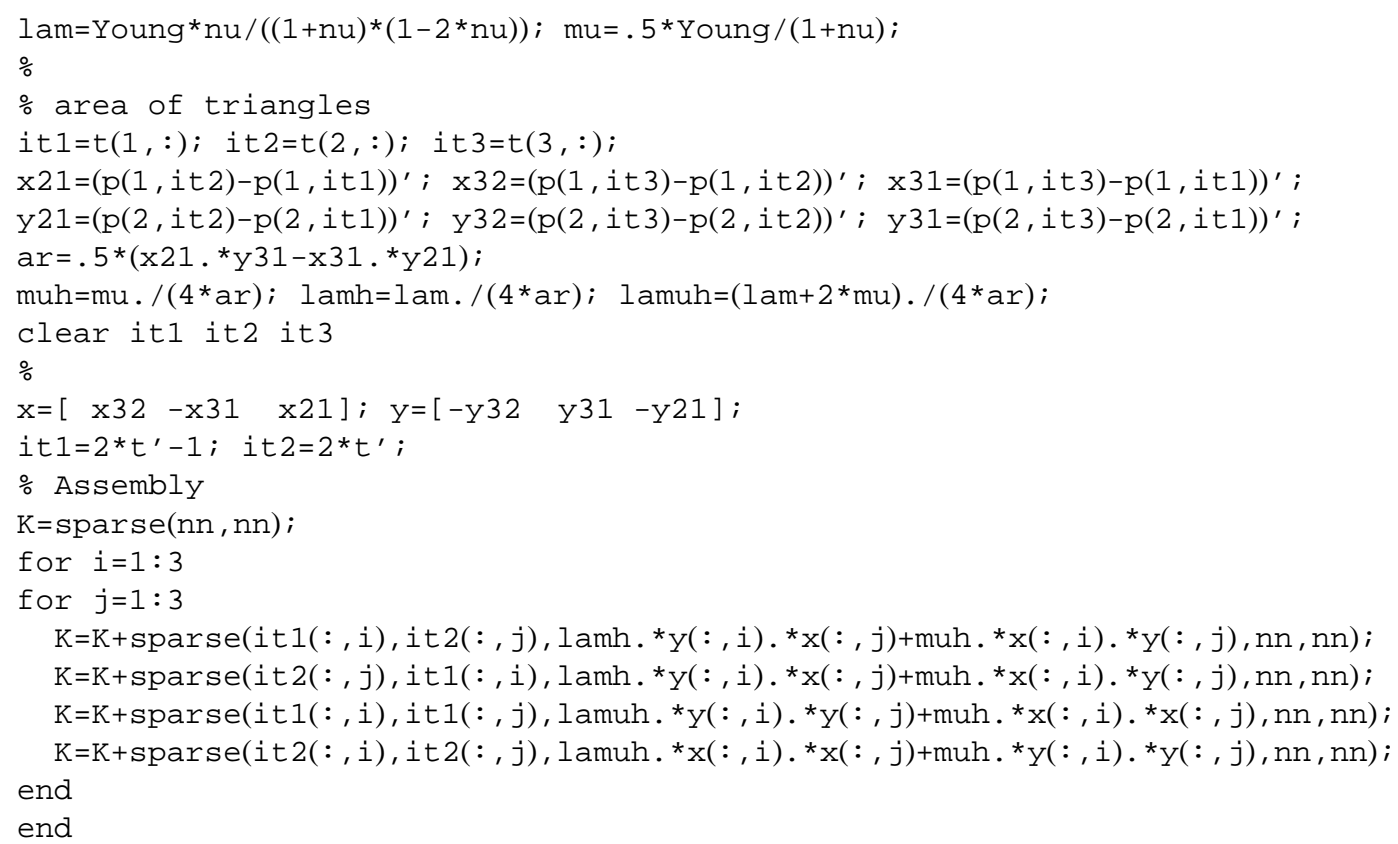

\section{Assembling the right-hand side}

We assume that the volume forces $f=\left(f_{1}, f_{2}\right)$ are provided at mesh nodes. The integral Eq. (10) is approximated as follows

$$
\int_{T} f \cdot \phi_{i} d x \approx \frac{1}{6} \operatorname{det}\left|\begin{array}{ll}
x_{2}-x_{1} & x_{3}-x_{1} \\
y_{2}-y_{1} & y_{3}-y_{1}
\end{array}\right| f\left(x_{c}, y_{c}\right), \quad j=\bmod (i-1,2)+1
$$

where $\left(x_{c}, y_{c}\right)$ is the center of mass of the triangle $T$. With the assumption on $f, f\left(x_{c}, y_{c}\right)=\left(f_{1}\left(x_{c}, y_{c}\right), f_{2}\left(x_{c}, y_{c}\right)\right)$ with

$$
f_{j}\left(x_{s}, y_{s}\right)=\left(f_{j}\left(x_{1}, y_{1}\right)+f_{j}\left(x_{2}, y_{2}\right)+f_{j}\left(x_{3}, y_{3}\right)\right) / 3, \quad j=1,2,
$$

where $\left\{\left(x_{i}, y_{i}\right)\right\}_{i=1,3}$ are vertices of the triangle $T$. Using the notation convention Eq. (14), we have

$$
\left|\begin{array}{cc}
x_{2}-x_{1} & x_{3}-x_{1} \\
y_{2}-y_{1} & y_{3}-y_{1}
\end{array}\right|=x_{21} y_{31}-x_{31} y_{12} \text {. }
$$

We can compute Eqs (18) and (19) over all triangles using vectorization techniques, and assemble the result with the Matlab function sparse. Matlab implementation of the assembly of the volume forces is presented in Function 3.

Function 3. Assembly of the right-hand side: body forces

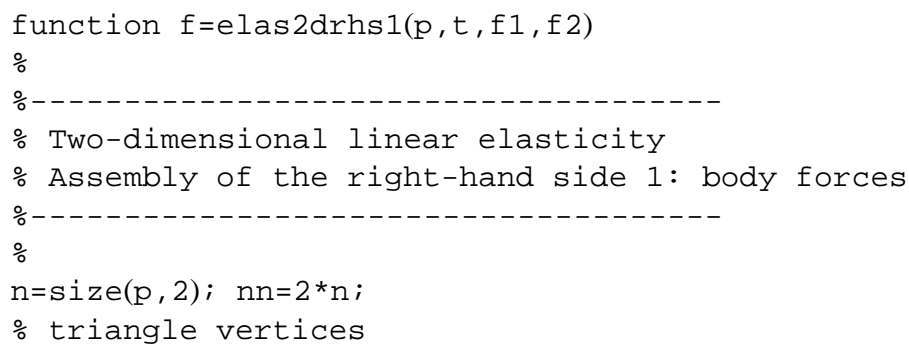




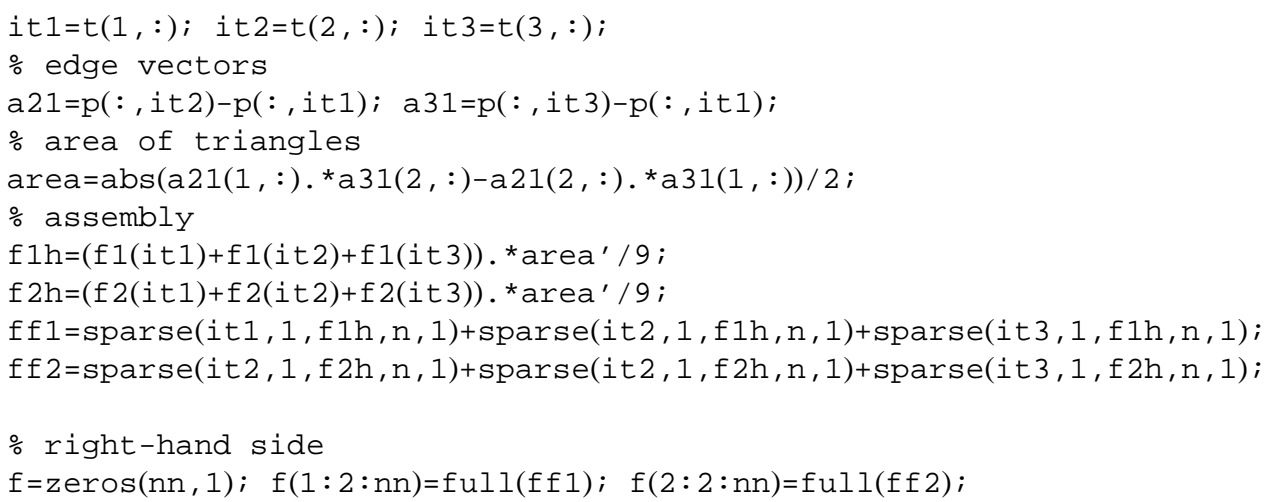

Integrals involving Neumann conditions are approximated using the value of $g=\left(g_{1}, g_{2}\right)$ at the center $\left(x_{c}, y_{c}\right)$ of the edge $E$

$$
\int_{E} g \cdot \phi_{i} d x \approx \frac{1}{2}|E| g\left(x_{c}, y_{c}\right)
$$

As for volume forces, $|E|$ and $g_{j}\left(x_{c}, y_{c}\right)$ are computed over all triangles using vectorization techniques and assembled using Matlab function sparse, Function 4.

Function 4. Assembly of the right-hand side: Neumann boundary conditions

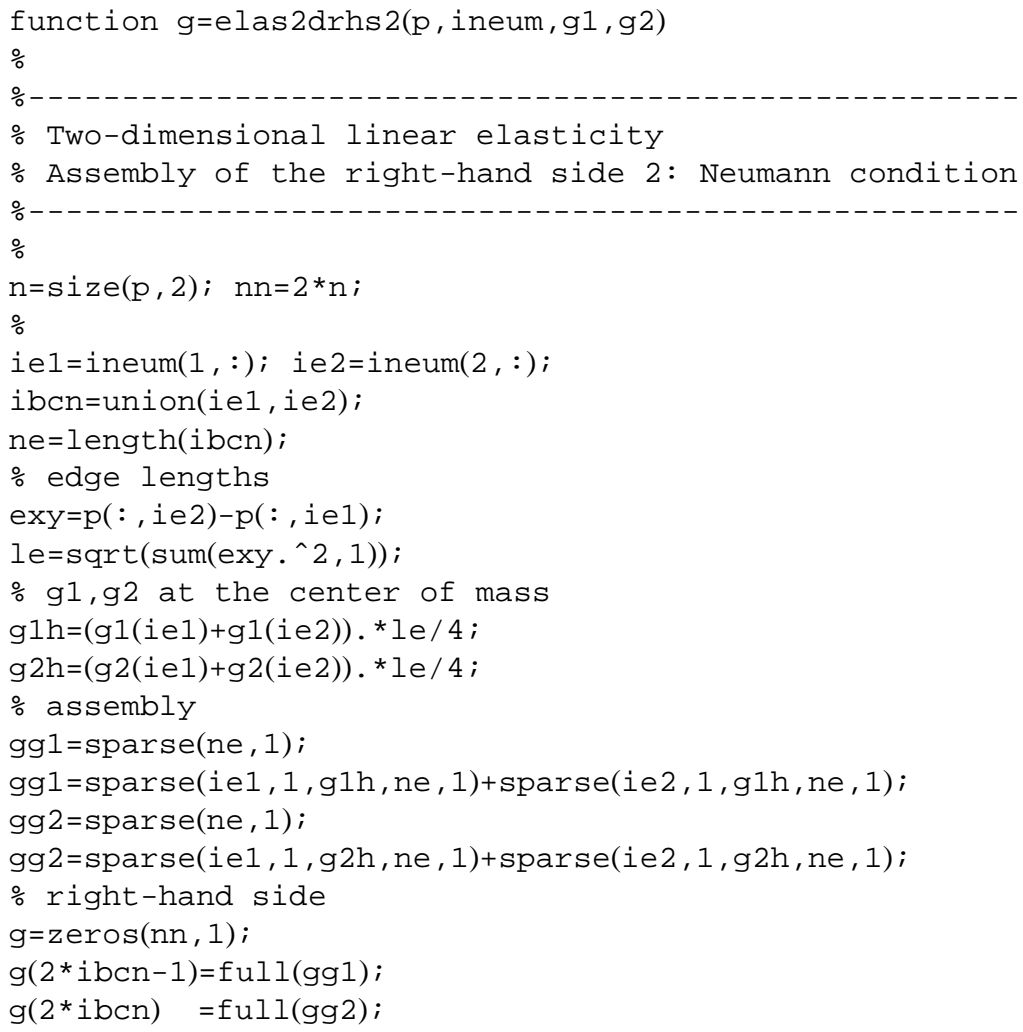




\section{Numerical experiments}

In elasticity, it is usual to display undeformed and deformed meshes. In the graphical representation of the deformed mesh, a magnification factor is used for the displacement. The Matlab Function 5 displays the deformed mesh with an additional function sf issued from post-processing. It is directly derived from the Matlab function Show given in [2]. The additional function can be stresses, strains, potential energy, etc. evaluated at mesh nodes.

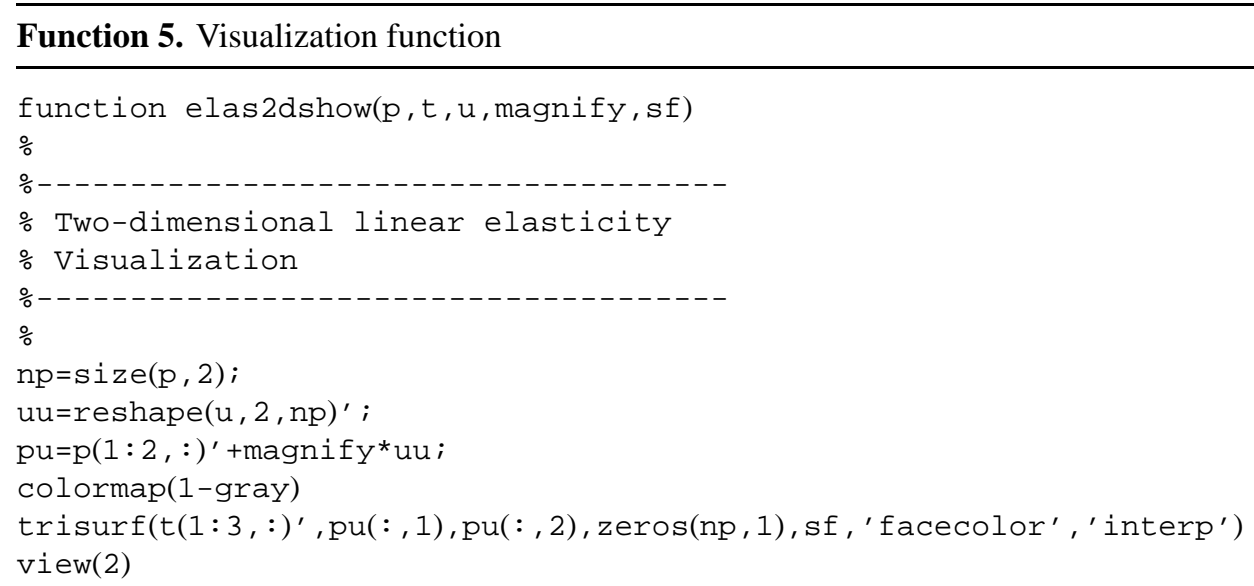

Function 6. Matlab function for computing the shear energy density Sed and the Von Mises effective stress Vms

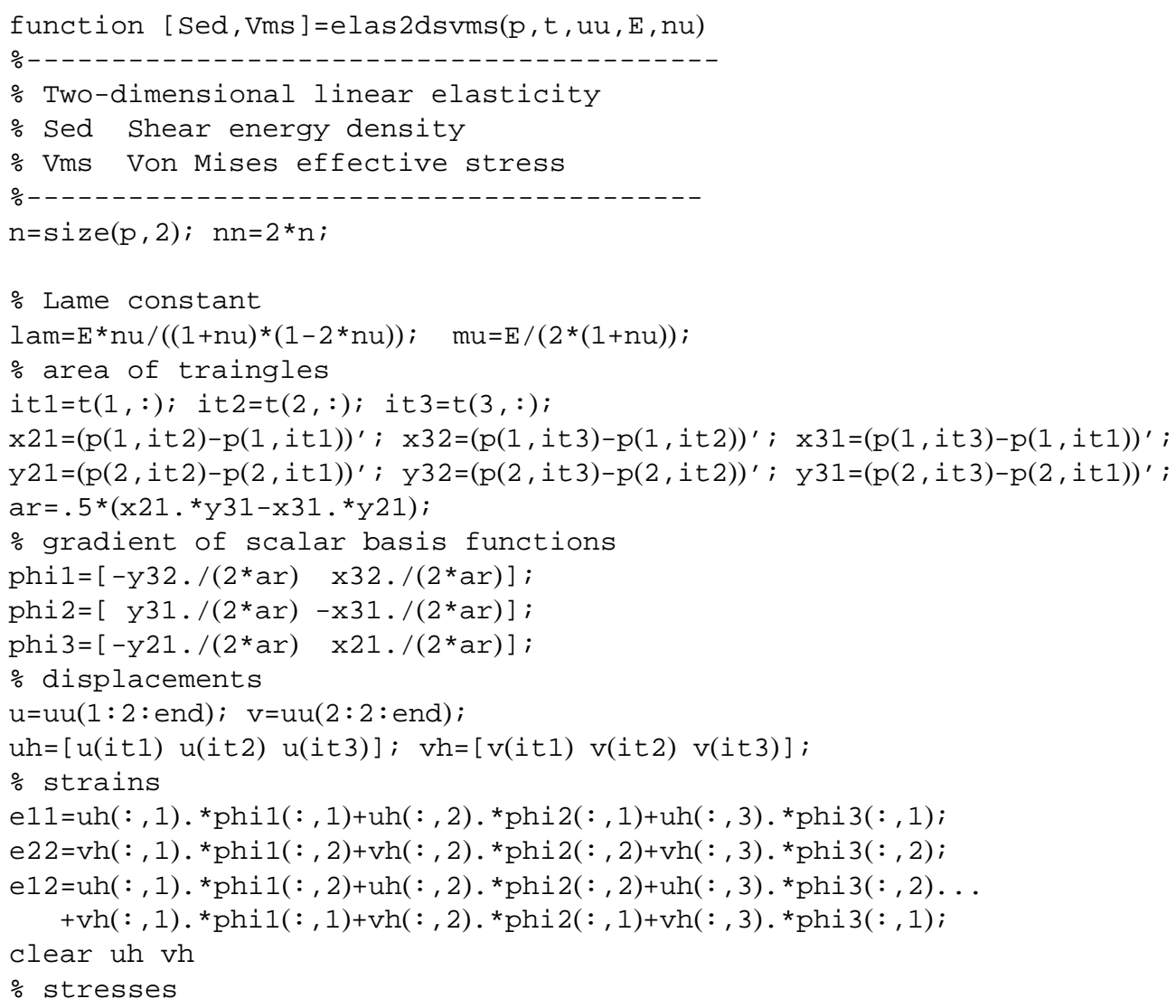




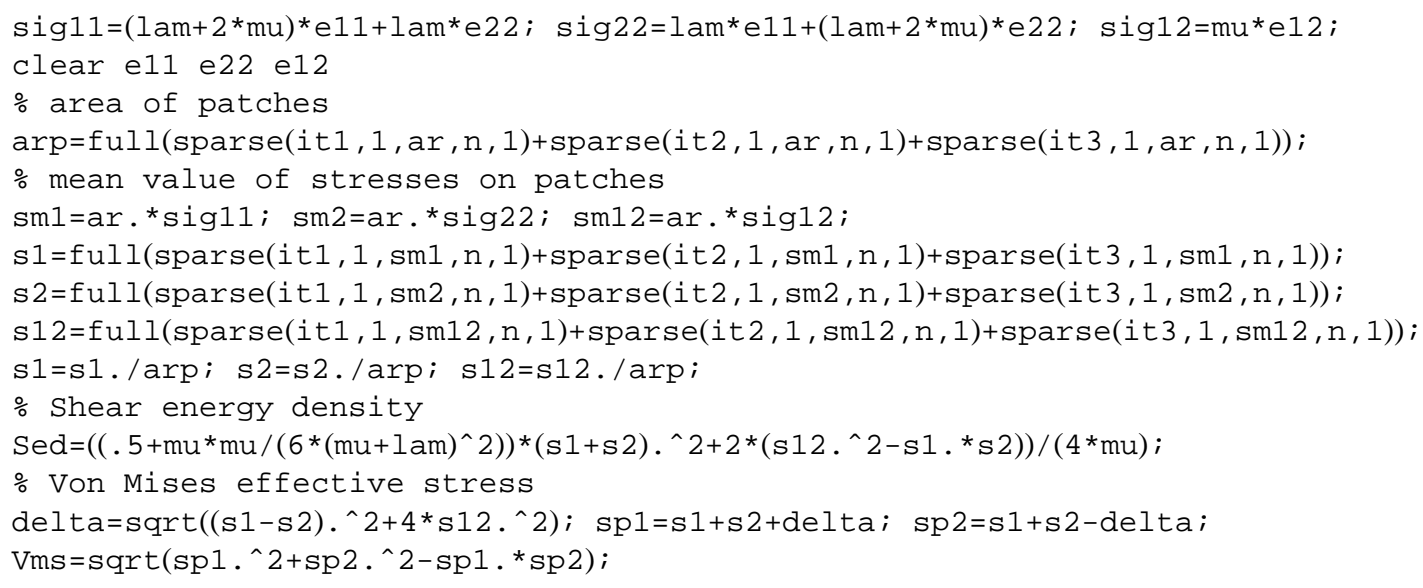

In our numerical experiments, the additional function used in the Function 5 is either the Von Mises effective stress or the shear energy density $\left|\operatorname{dev} \sigma_{h}\right|^{2} /(4 \mu)$, where

$$
\left|\operatorname{dev} \sigma_{h}\right|^{2}=\left(\frac{1}{2}+\frac{\mu^{2}}{6(\mu+\lambda)}\right)\left(\sigma_{h 11}+\sigma_{h 22}\right)^{2}+2\left(\sigma_{h 12}^{2}-\sigma_{h 11} \sigma_{h 22}\right) .
$$

The stress tensor $\sigma_{h}$ is computed at every node as the mean value of the stress on the corresponding patch. Matlab Function 6 calculates approximate shear energy density and Von Mises effective stress.

In all examples, Dirichlet boundary conditions are taken into account by using large spring constants (i.e. penalization).

\subsection{L-shape}

The L-shape test problem is a common benchmark problem for which the exact solution is known. The domain $\Omega$ is described by the polygon

$$
(-1,-1),(0,-2),(2,0),(0,2),(-1,-1),(0,0) .
$$

The exact solution is known in polar coordinates $(r, \theta)$,

$$
\begin{aligned}
& u_{r}(r, \theta)=\frac{1}{2 \mu} r^{\alpha}\left[\left(c_{2}-\alpha-1\right) c_{1} \cos ((\alpha-1) \theta)-(\alpha+1) \cos ((\alpha+1) \theta)\right], \\
& u_{\theta}(r, \theta)=\frac{1}{2 \mu} r^{\alpha}\left[(\alpha+1) \sin ((\alpha+1) \theta)+\left(c_{2}+\alpha-1\right) c_{1} \sin ((\alpha-1) \theta)\right] .
\end{aligned}
$$

The exponent $\alpha$ is the solution of the equation

$$
\alpha \sin (2 \omega)+\sin (2 \omega \alpha)=0
$$

with $\omega=3 \pi / 4$ and

$$
c_{1}=-\frac{\cos ((\alpha+1) \omega)}{\cos ((\alpha-1) \omega)}, \quad c_{2}=2 \frac{\lambda+2 \mu}{\lambda+\mu} .
$$

The displacement field Eqs (20)-(21) solves Eqs (2)-(4) with $f=0$ and $u_{D}=\left(u_{r}, u_{\theta}\right)$ on $\Gamma=\Gamma_{D}$. Numerical experiments are carried out with Young's modulus $E=100000$ and Poisson's coefficient $\nu=0.3$. The magnitude of the gradient $|\nabla u|$ of the exact solution Eqs (20)-(21) has a singularity at the re-entrant corner $(0,0)$. This singularity, despite being very local, is a significant source of error.

To know the percentage of computing effort the assembling functions take, we have run the Matlab program given in Appendix (without the four last lines) with the Matlab command profile which records informations about execution time, number of calls, parent functions, child functions, code line hit count, etc. Figures 2-3 
Table 1

Percentage of computing effort taken by elas 2 dmat 1

\begin{tabular}{lcccccc}
\hline Mesh triangles & 100 & 400 & 1600 & 6400 & 25600 & 102400 \\
Percentage of CPU & $57.1 \%$ & $76.2 \%$ & $90.5 \%$ & $95.8 \%$ & $98.9 \%$ & $99.7 \%$ \\
\hline
\end{tabular}

Table 2

Performances of assembling functions elas 2 dmat 1 and elas 2 dmat 2

\begin{tabular}{lcc}
\hline \multirow{2}{*}{$\begin{array}{l}\text { Mesh } \\
\text { triangles/nodes }\end{array}$} & \multicolumn{2}{c}{ CPU time (in Sec.) } \\
\cline { 2 - 3 } & elas2dmat1 & elas2dmat2 \\
\hline $100 / 66$ & 0.04 & 0.02 \\
$400 / 231$ & 0.16 & 0.03 \\
$1600 / 861$ & 0.86 & 0.11 \\
$6400 / 3321$ & 7.95 & 0.48 \\
$25600 / 13041$ & 148.02 & 2.13 \\
$102400 / 51681$ & 3394.51 & 8.95 \\
\hline
\end{tabular}

Table 3

Percentage of computing effort taken by elas 2 dmat 2

\begin{tabular}{lcccccc}
\hline Mesh triangles & 100 & 400 & 1600 & 6400 & 25600 & 102400 \\
Percentage of CPU & $40.0 \%$ & $42.0 \%$ & $50.0 \%$ & $57.0 \%$ & $57.0 \%$ & $49.7 \%$ \\
\hline
\end{tabular}

\begin{tabular}{|c|c|c|c|c|}
\hline Eunction Name & Calls & $\begin{array}{l}\text { Total } \\
\text { Time }\end{array}$ & Sime & $\begin{array}{l}\text { Total Time } \\
\text { Plot } \\
\text { (dark band } \\
\text { - seit time) }\end{array}$ \\
\hline elaszdoxole & 1 & $\begin{array}{l}149.680 \\
=\end{array}$ & 1. $600 \mathrm{~s}$ & 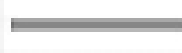 \\
\hline elaszamat1. & 1 & $\begin{array}{l}147.990 \\
s\end{array}$ & $\begin{array}{l}147,900 \\
=\end{array}$ & \\
\hline $\begin{array}{l}\text { mpower } \\
\text { (Duiltin-fu nction) }\end{array}$ & 1 & $0=$ & $0.000=$ & \\
\hline cartepas & 1 & 06 & 0.0006 & \\
\hline enere & 1 & $0=$ & $0.000=$ & \\
\hline $\begin{array}{l}\text { display } \\
\text { (Buitin-nu netion) }\end{array}$ & 1 & 0 s & o.000 s & \\
\hline
\end{tabular}

Self time in the time mpent in a function excluding the time spent in its ehill functions. Belf time also includes overtead resulting from the prosess of protiting

Fig. 2. Matlab profile command report with elas 2 dmat 1 assembling function using a mesh with 13041 nodes.

show informations recorded with a mesh with 13041 nodes. It appears that the assembling function elas $2 \mathrm{dmat} 1$ takes about $98 \%$ of CPU time. Note that the time given in Table 3 for the assembling operations is the time taken by intruction $\mathrm{K}=\mathrm{elas} 2 \mathrm{dmat} 1(\mathrm{p}, \mathrm{t}, \mathrm{E}, \mathrm{nu})$ while the time given in Table 2 is only the time spent in the assembling function (without calling and returning operations). Table 1 shows clearly that the assembling function elas 2 dmat 1 is the bottleneck of the program.

We now compare the performances of Matlab functions elas 2 dmat 1 and elas 2 dmat 2 for assembling the stiffness matrix of the L-shape problem. To this end, various meshes of the L-shape are generated and we use Matlab command profile to compute the elapsed time. Table 2 shows the performances of assembling Functions 1-2. One can notice that the saving of computational time, with the vectorized function elas 2 dmat 2 , is considerable. Table 3 shows that the finite element program with elas 2 dmat 2 is more balanced compared to elas $2 \mathrm{dmat} 1$.

We report in Table 4 the $H^{1}$ and $L^{2}$ distances between the exact solution Eqs (20)-(21) and the approximate solution using the assembling function elas 2 dmat2. The distances are computed using a 13-point Gaussian quadrature. One can notice that $\left\|u-u_{h}\right\|_{L^{2}(\Omega)} \rightarrow 0$ and $\left\|u-u_{h}\right\|_{H^{1}(\Omega)} \rightarrow 0$ has the mesh size $h$ goes to zero but the convergence rates are lower than theoretical ones ( 2 for the $L^{2}$-error and 1 for the $H^{2}$-error for elliptic problems, 
Table 4

\begin{tabular}{|c|c|c|c|c|}
\hline Mesh nodes & $\left\|u-u_{h}\right\|_{L^{2}(\Omega)}$ & Rate & $\left\|u-u_{h}\right\|_{H^{1}(\Omega)}$ & Rate \\
\hline 66 & $1.7843 \times 10^{-6}$ & & $1.7372 \times 10^{-5}$ & \\
\hline 231 & $7.1572 \times 10^{-7}$ & 1.31 & $1.2058 \times 10^{-5}$ & 0.52 \\
\hline 861 & $2.8760 \times 10^{-7}$ & 1.31 & $8.3279 \times 10^{-6}$ & 0.53 \\
\hline 3321 & $1.1624 \times 10^{-7}$ & 1.30 & $5.7377 \times 10^{-6}$ & 0.53 \\
\hline 13041 & $4.7203 \times 10^{-8}$ & 1.30 & $3.9449 \times 10^{-6}$ & 0.54 \\
\hline 51681 & $1.9220 \times 10^{-8}$ & 1.29 & $2.7089 \times 10^{-6}$ & 0.54 \\
\hline
\end{tabular}

\begin{tabular}{|c|c|c|c|c|c|}
\hline \multicolumn{6}{|c|}{ Lines where the most trme was spent } \\
\hline LineNarber & Code & Cals & Fotd fine & * Tine & fineflot \\
\hline 43 & 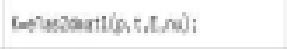 & 1 & 148.0005 & $96.5 \%$ & \\
\hline 55 & walt; & 1 & $2.4: 05$ & $0.9 \%$ & 1 \\
\hline 51 & 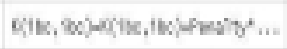 & 1 & 02105 & Q.1\% & \\
\hline 29 & 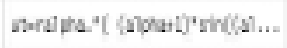 & 1 & Q020.0s & Q.心 & \\
\hline 26 & nilptor.welpavena); & 1 & 0.0005 & tod & \\
\hline Ctter Ines sै owshead & & & 000205 & ack & \\
\hline Totali & & & $149.680 \mathrm{~s}$ & $100 \%$ & \\
\hline
\end{tabular}

Fig. 3. Matlab profile command report details with elas 2 dmat 1 assembling function using a mesh with 13041 nodes.

see e.g. $[3,4])$. The reason is that the exact solution $u$ does not belong to $H^{2}(\Omega)$ so that the error estimate theorems do not hold.

Figure 4 shows the deformed mesh (231 nodes and 400 triangles) of the L-shape with a displacement field multiplied by a factor 3000 . The grey tones visualize the approximate shear energy density and show the singularity at $(0,0)$.

\subsection{A membrane problem}

We consider a two-dimensional membrane $\Omega$ described by the polygon

$$
(0,-5),(35,-2),(35,2),(0,5)
$$

with $E=30000$ and $\nu=0.4$. The membrane is clamped at $x=0$ and subjected to a a volume force $f=(0,-0.75)$ and shearing load $g=(0,10)$ at $x=35$. Figures 5-6 show initial (undeformed) and deformed configurations of the membrane, for a mesh with 995 nodes (i.e. 1990 degrees of freedom). The magnification factor, for the displacement field, is 20. The grey tones visualize the approximate Von Mises effective stress. The deformed configuration shows peak stresses at corners $(0,-5)$ and $(0,5)$ as expected.

\section{Conclusion}

We have demonstrated that, for solving isotropic linear elasticity problems in Matlab with the finite element method, the vectorized code is much more efficient than a standard implementation with a loop over triangles.

Further work is underway to derive vectorized codes for the three-dimensional linear elasticity using tetrahedral elements. The main difficulty is to invert analytically, with easy to implement formulas, the matrix 


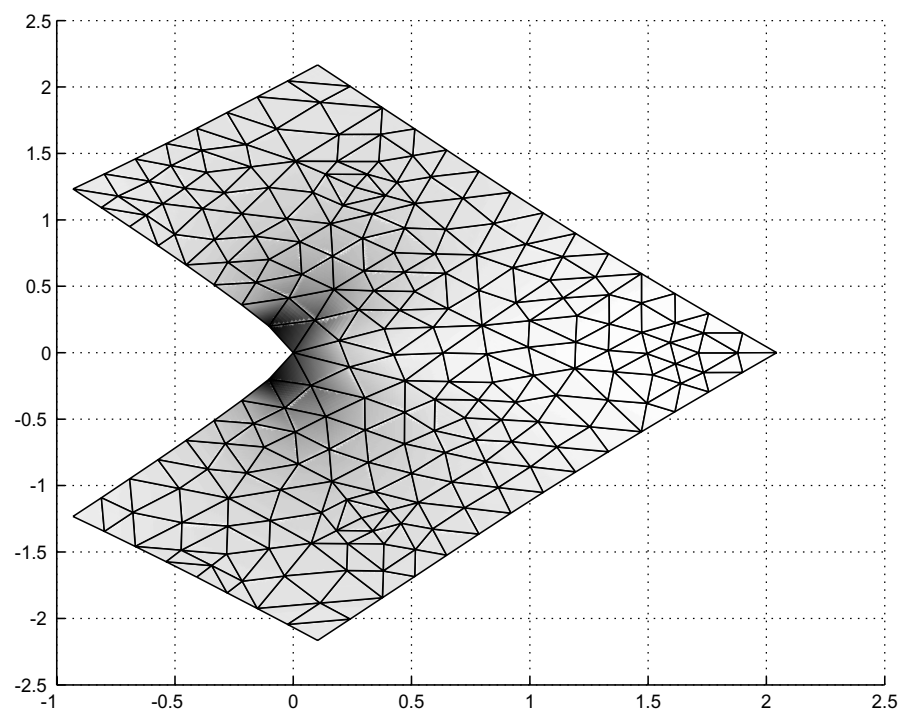

Fig. 4. Deformed mesh for the L-shape problem.

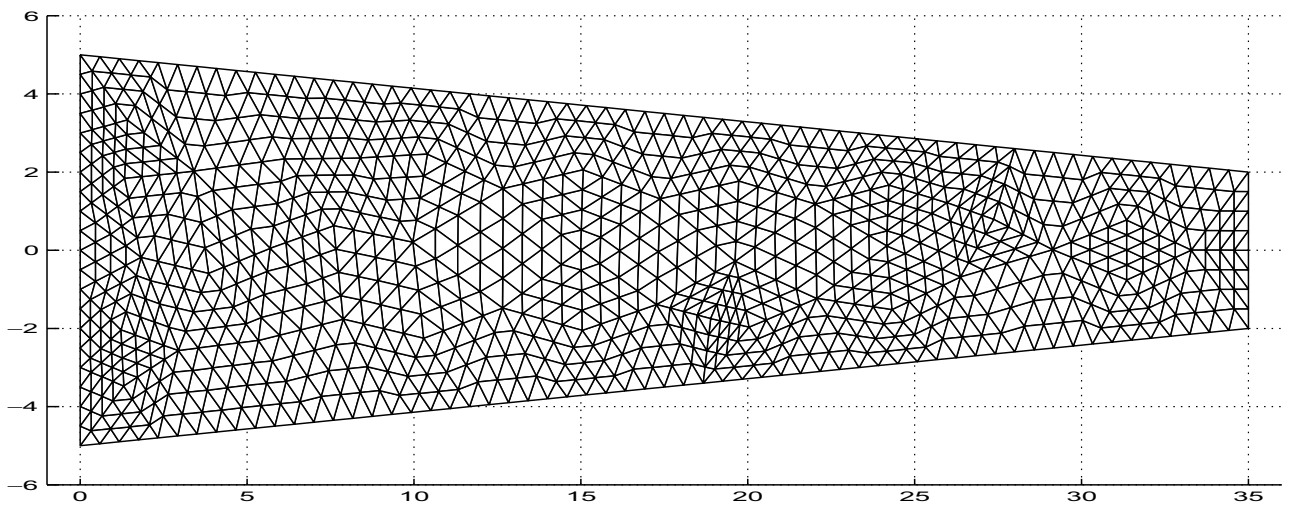

Fig. 5. Elastic Membrane: Initial configuration.

$$
\left[\begin{array}{cccc}
1 & 1 & 1 & 1 \\
x_{1} & x_{2} & x_{3} & x_{4} \\
y_{1} & y_{2} & y_{3} & y_{4} \\
z_{1} & z_{2} & z_{3} & z_{4}
\end{array}\right]
$$

where $\left\{\left(x_{i}, y_{i}, z_{i}\right)\right\}_{i=1, \ldots, 4}$ are the vertices of a tetrahedron. Indeed, if $\left\{\varphi_{i}\right\}_{i}$ are the linear basis functions on a tetrahedron, their gradients are given by (see e.g. [2])

$$
\left[\begin{array}{l}
\nabla \varphi_{1}^{t} \\
\nabla \varphi_{2}^{t} \\
\nabla \varphi_{3}^{t} \\
\nabla \varphi_{4}^{t}
\end{array}\right]=\left[\begin{array}{cccc}
1 & 1 & 1 & 1 \\
x_{1} & x_{2} & x_{3} & x_{4} \\
y_{1} & y_{2} & y_{3} & y_{4} \\
z_{1} & z_{2} & z_{3} & z_{4}
\end{array}\right]^{-1}\left[\begin{array}{lll}
0 & 0 & 0 \\
1 & 0 & 0 \\
0 & 1 & 0 \\
0 & 0 & 1
\end{array}\right]
$$




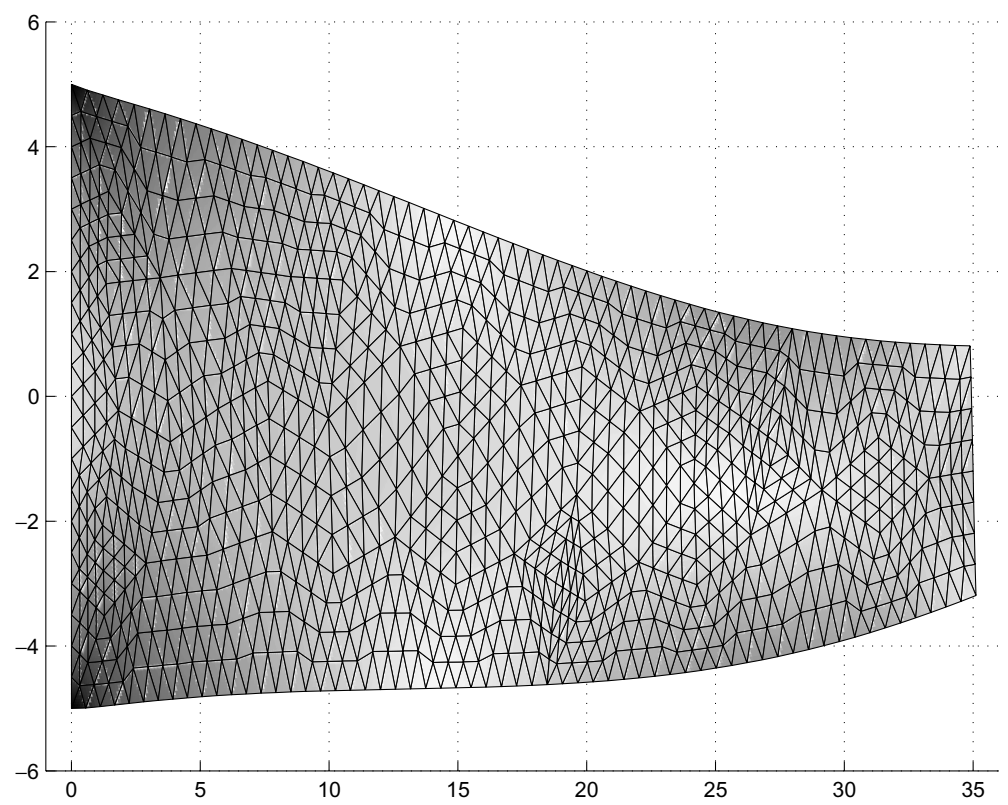

Fig. 6. Elastic membrane: deformed configuration.

\section{Appendix}

Main program for the L-shape problem

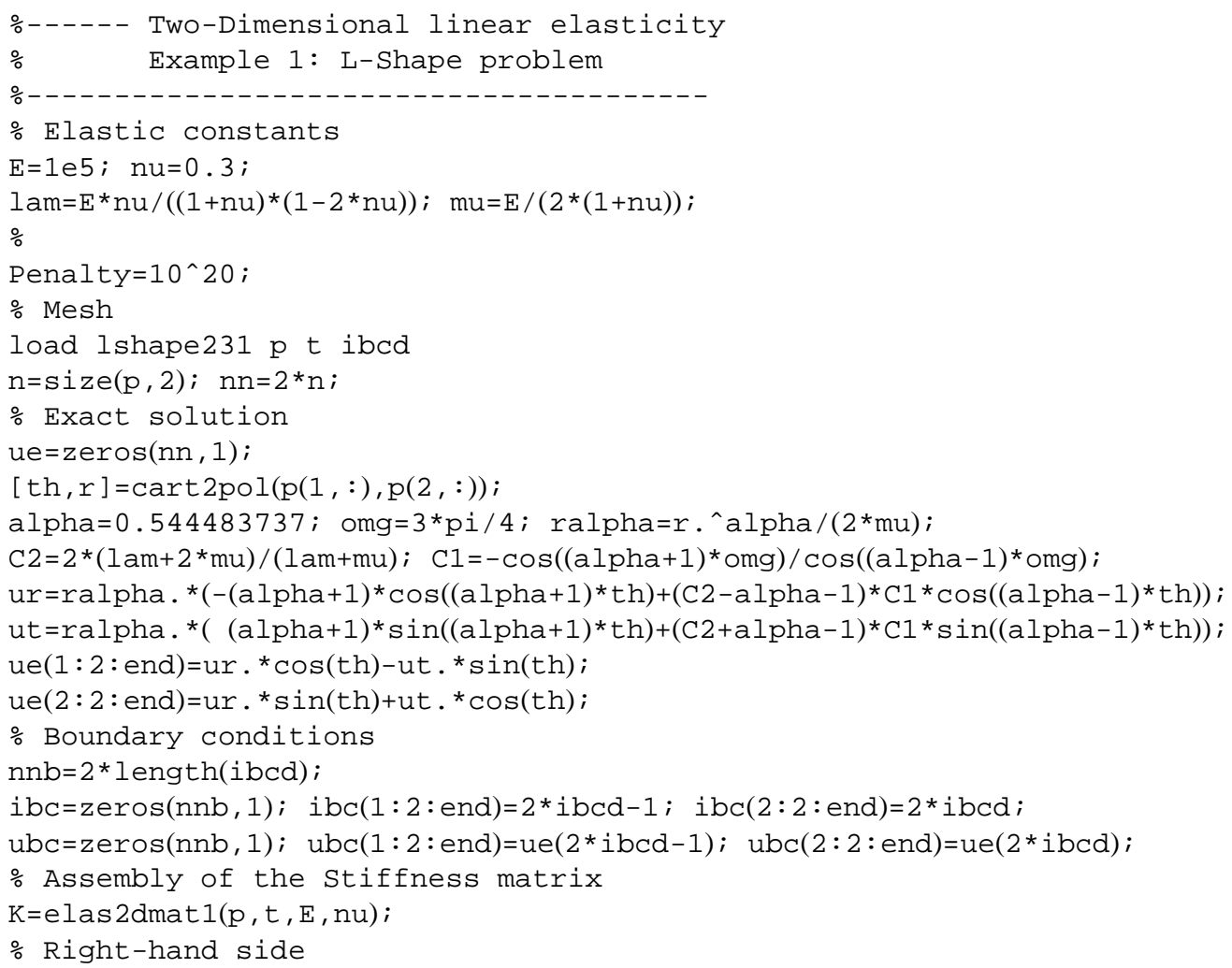




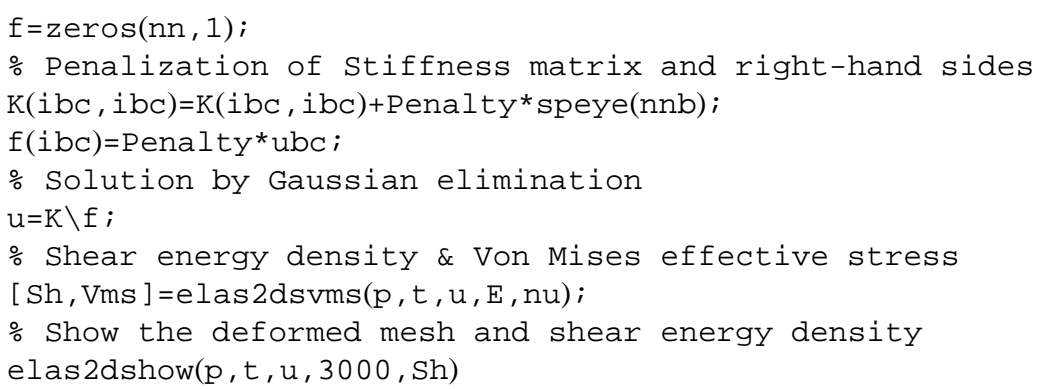

\section{Main program for the membrane problem}

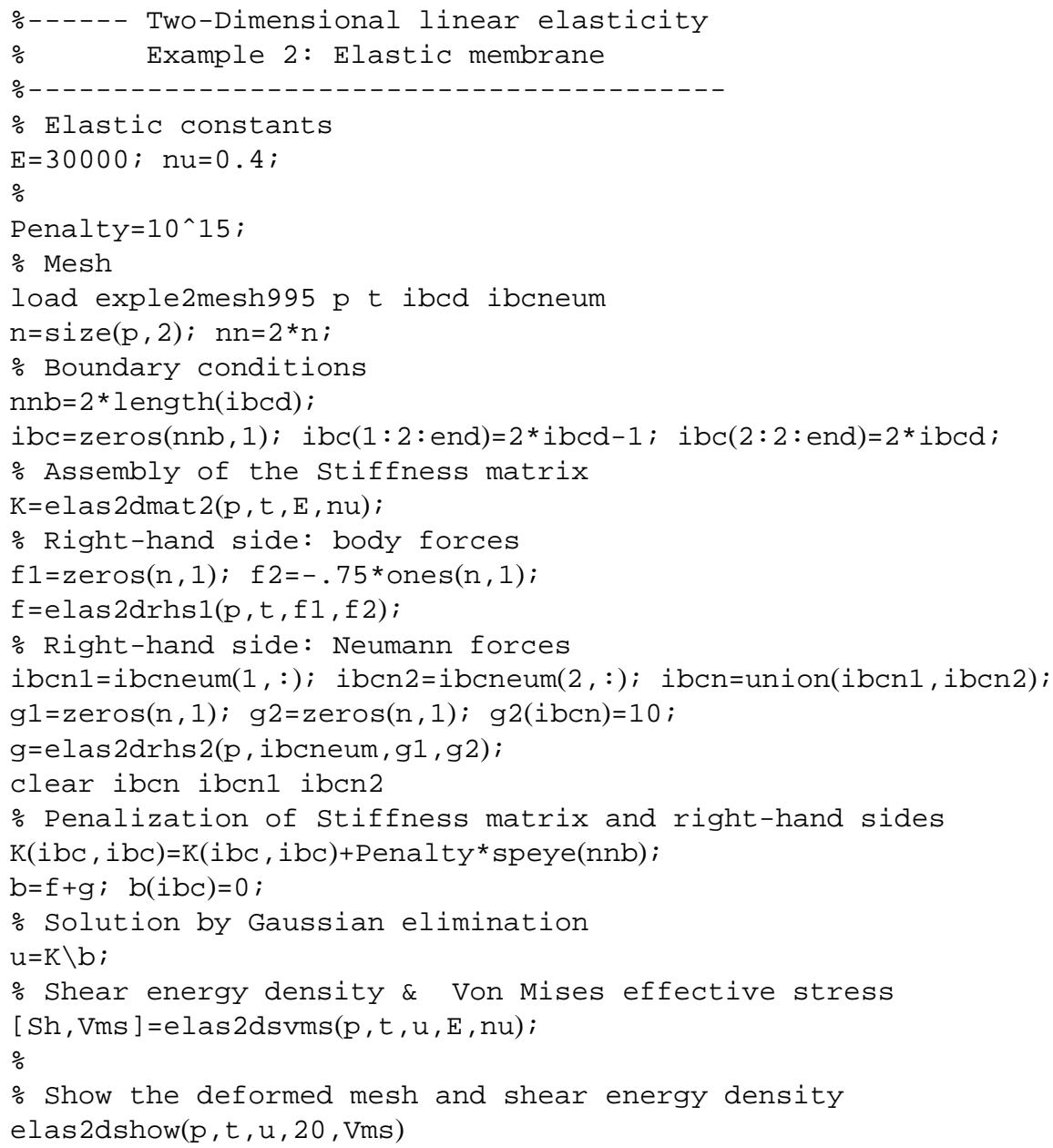

\section{References}

[1] J. Alberty, C. Carstensen and S.A. Funken, Remarks around 50 lines of matlab: short finite element implementation, Numer Algorithms 20 (1999), 117-137.

[2] J. Alberty, C. Carstensen, S.A. Funken and R. Klose, Matlab implementation of the finite element method in elasticity, Computing 69 (2002), 239-263.

[3] P.G. Ciarlet, The Finite Element Method for Elliptic Problems, North-Holland, Amsterdam, 1979. 
[4] P.-G. Ciarlet, Basic error estimates for elliptic problems, in: Finite Element Methods (Part 1), P.-G. Ciarlert and J.-L. Lions, eds, volume II of Handbook of Numerical Analysis, North-Holland, Amsterdam, 1991, pp. 23-343.

[5] Y.W. Kwon and H. Bang, The Finite Element Method Using MATLAB, CRC Press, New York, 2000

[6] L. Langemyr, A. Nordmark, M. Ringh, A. Ruhe, Oppelstrup and M. Doro-Bantu, Partial Differential Equations Toolbox User's Guide, The Math Works, Inc., 1995

[7] P.-O. Persson and G. Strang, A simple mesh generator in Matlab, SIAM Rev 42 (2004), 329-345. 

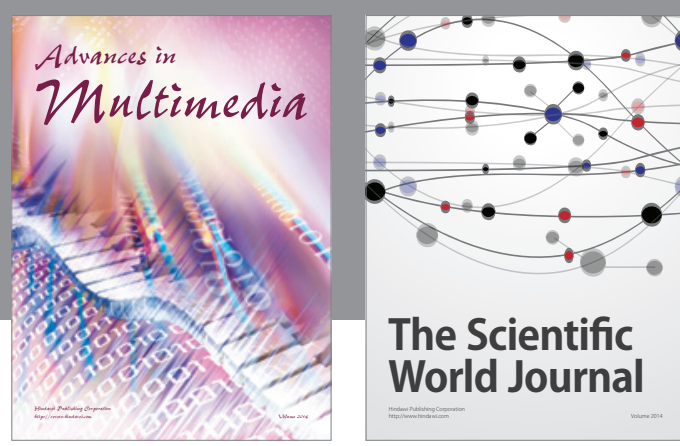

The Scientific World Journal
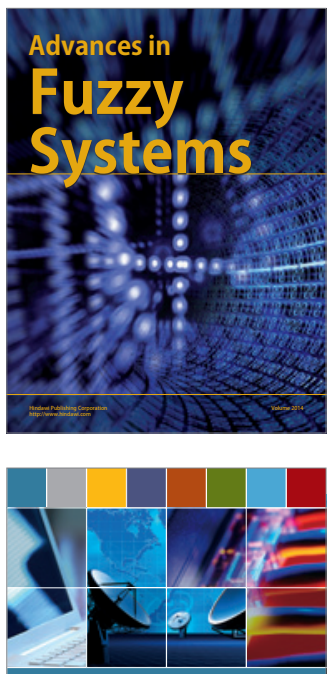

Computer Networks and Communications
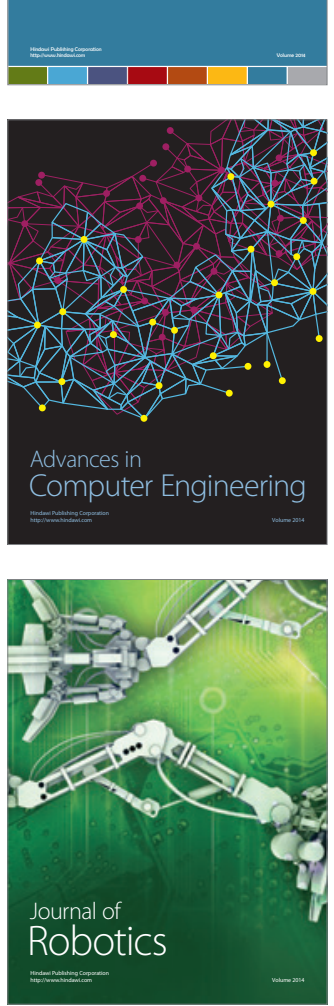
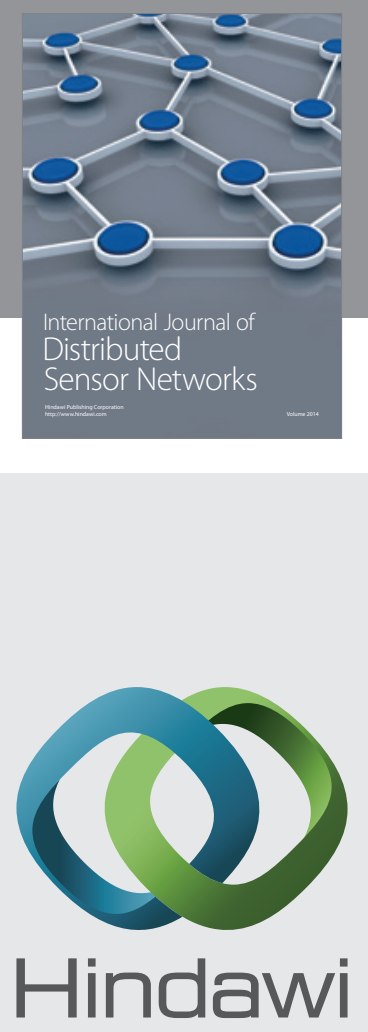

Submit your manuscripts at

http://www.hindawi.com
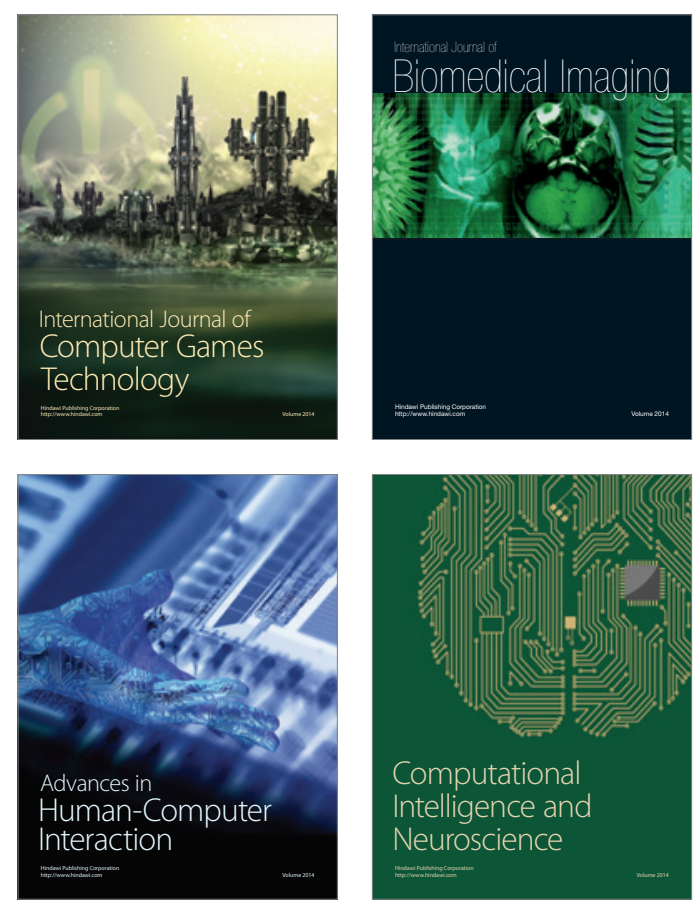
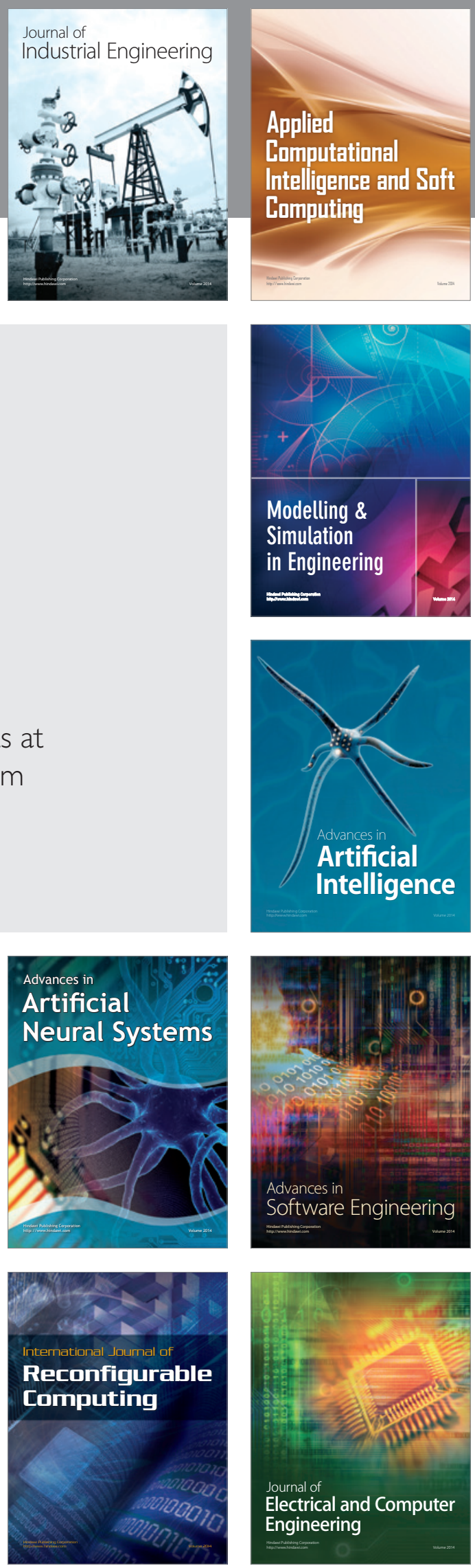